\title{
Introduction to the Special Issue on Shared Control: Applications
}

\author{
Mark Mulder, David A. Abbink \\ Delft University of Technology \\ and \\ Tom Carlson \\ University College London
}

\begin{abstract}
Shared control is an exciting up-and-coming engineering field that is blending the boundaries of control, where humans interact with robots or vehicles that are partly automated. The main challenges for robotics and automation today are posed by the less structured, unpredictable environments, in which humans operate naturally, especially when they need to interact with other humans. As a result, many semi-automated systems today need to be supervised by human operators, but as fast as the levels of automation of many systems are increasing, we also need to speed up how we think about what this implies for human-machine interaction. The widely applied paradigm of human-centered automation has been popular and useful for the past two decades, but it requires an update with the current trend in automation becoming more ubiquitous in human environments and less dependent on explicit input from the human operator. In contrast to the supervisory control paradigm - where control is traded between human and machine- the shared control paradigm implicitly assumes the interaction between two or more independent agents that together perform a task to achieve a common goal. This implies that the design of the shared control system is not necessarily only human centered. In shared control systems, all the acting agents need to be aware of the others' capabilities, weaknesses, and authority. Hence, reciprocal communication of each agent's operational boundaries, whether human or machine, is essential.

This Special Issue of the Journal of Human-Robot Interaction (JHRI) is dedicated to showing the wide variety of applications where the ideas of shared control are currently being applied. We solicited papers from diverse fields of engineering, which resulted in topics ranging from vehicle control to rehabilitation robots and even to designing human-robot dialogue systems. The breadth of subjects where the shared control paradigm is applicable shows its potential to advance the human-centered control paradigm. The challenge, now, is to learn from each other and discover whether we can formulate a comprehensive taxonomy of shared control inclusive of all these different applications while creating a more unified approach to designing and evaluating shared control systems that enables the responsible and engaging human-machine interaction required for the coming decades.

The first two articles in this special issue present shared control designs for the automotive domain, which is currently struggling with the open question of how to responsibly and intuitively couple the driver to the abilities of highly automated vehicles. First, Makoto Itoh, Hiroto Tanaka,
\end{abstract}

\footnotetext{
Authors retain copyright and grant the Journal of Human-Robot Interaction right of first publication with the work simultaneously licensed under a Creative Commons Attribution License that allows others to share the work with an acknowledgement of the work's authorship and initial publication in this journal.
} 
and Toshiyuki Inagaki present a shared control design for pedestrian collision avoidance based on haptics (the sense of touch). Their automation system provides the driver with a steering torque to help steer the vehicle away from an unexpected crossing pedestrian, while keeping the driver in the direct control loop. The results of their experiment with a driving simulator showed that the reaction time of drivers was reduced significantly, and that the system was effective to enhance driver's appropriate selection of steering direction in many cases - but not in all. Hence, further research is required to understand how to better match the support system's initial steering direction and the corresponding driver reaction.

This is followed well by Ryota Nishimura, Takahiro Wada, and Seiji Sugiyama who describe a haptic shared control system for steering a vehicle. Their contribution aimed to understand how to communicate differences in desired trajectories between driver and automation system: how to support drivers in maintaining lane-position, while also allowing for smooth lane-changes? They proposed to integrate these contradicting driving conditions by evaluating the cooperative status of haptic shared control between the operator and the automated system based on two axes: the initiative holder and intent consistency between the two agents. The results of the presented experiment demonstrate the effectiveness of their proposed method.

The third paper, by Michael Dorneich, Emmanuel Letsu-Dake, Sanjiv Singh, Sebastian Scherer, Lyle Chamberlain, and Marcel Bergerman, shows how complex vehicle control-in this case a roadable air vehicle-can be facilitated with shared control. The results of their experiment suggest that a mixed-initiative paradigm leverages the benefits of human and automated control, while avoiding the drawbacks associated with each.

Moving away from the vehicular applications in the fourth paper, Ramtin Rakhsha and Daniela Constantinescu discuss the complexity of multi-agent interaction in a distributed network and show how such interactions can be improved by applying their average position (AP) strategy in controlling the stiffness of the shared virtual object (SVO). This paper shows that AP coordination has bounded effective coordination gain and increases the stability region of distributed multi-user haptic cooperation, as compared to conventional proportional-derivative (PD) coordination. This resulted in participants reporting a more natural-feeling interaction, with less viscous SVO dynamics, as compared with PD coordination.

The fifth paper, by Harold Soh and Yiannis Demiris, introduces a framework, probabilistic model, and algorithm for learning shared control policies by observing an assistant-a methodology they refer to as Learning Assistance by Demonstration (LAD). Applying this methodology to assisting a wheelchair user, they demonstrate that their learned shared control policy improved driving performance with a speedup of $191 \%$. Wheelchair users also felt better able to complete the prescribed course in the laboratory.

Yoshiyuki Tanaka then shows, in the sixth paper, that a robot-aided training methodology focusing on smooth transient trajectory generation by the arm can accelerate the learning of motor functions. He applies the methodology to a virtual curling task and shows that participants were able to learn highly specialized smooth hand movements. This kind of training is pertinent to stroke patients who are unable to perform natural movements, and hence, require repetitive motor rehabilitation. The presented method was shown to help in recovering the trainee's functions of motor control and learning.

An entirely different but equally interesting topic is presented by Sklar and Azhar. They apply the shared control paradigm to the application of argumentation-based dialogue games as a means to facilitate flexible human-robot social interaction, including unscripted changes in initiative. They describe a methodology for implementing multiple types of argumentation-based dialogues for human-robot interaction and give examples defined in the test environment, 'Treasure Hunt Game.'

Shared control between human and robot can be made possible by robotic interfaces. The eighth paper, by Mark Fehlberg, Hamidreza Sani, and William Provancher, describes the design of a planar active handrest (PAHR) that improves user precision manipulation. Three experiments 
evaluated controller designs to select the most effective method to control the device. The authors conclude that a rotational degree of freedom (DOF) allows the enhanced PAHR to better follow the kinematics of a user's planar arm movements while allowing skill level equal to the PAHR, with reduced user force input and lower perceived exertion.

Finally, in the ninth paper, Antonio Moualeu and Jun Ueda propose a method for predicting the endpoint stiffness of a human operators' limb. Knowledge of this adaptive human property is essential to optimally tune forces generated by joysticks or steering wheels in the haptic shared control paradigm. The proposed methodology is based on measured co-contraction levels of a select group of muscles of the operator. It incorporates an upper extremity musculoskeletal model that accounts for muscle redundancy and the role of muscle co-contraction on arm stiffness modulation. The ultimate goals of this research are to understand the relationships between neuromuscular adaptations and performance characteristics in physical human-robot interaction, in order to develop and validate a novel haptic control system that tunes its gains according to stochastic changes in operator physical and cognitive state.

These nine papers constitute an overview of the broad applicability of the shared control paradigm. We hope that these studies provide fresh insight both to those new to this field and to established experts looking for the latest advances in the design and evaluation of shared control systems that allow mutual interaction between human and machine.

Mark, David, and Tom

\section{Acknowledgements}

We would like to thank the Journal of Human-Robot Interaction team, especially Jeanie Lyubelsky and Mike Goodrich, as well as all the reviewers for their contributions to making this special issue possible. Our ideas for this special issue were triggered and stimulated by the ongoing discussions and collaborations stimulated by the IEEE Systems, Man, and Cybernetics' Technical Committee on Shared Control, which aims to bring together researchers and research in the field of shared control.

Guest editors' names and contact information: Mark Mulder, Delft University of Technology, Faculty $3 \mathrm{mE}$, Department of BioMechanical Engineering, Delft Haptics Lab, Delft, The Netherlands. Mark.Mulder@tudelft.nl; David A. Abbink, Delft University of Technology, Faculty $3 \mathrm{mE}$, Department of BioMechanical Engineering, Delft Haptics Lab, Delft, The Netherlands. D.A.Abbink@tudelft.nl; Tom Carlson, University College London, Aspire Centre for Rehabilitation Engineering and Assistive Technology, London, UK. T.Carlson@ucl.ac.uk. 